\title{
Energy-efficient user grouping algorithms for power minimisation in multi-carrier code division multiple access systems
}

\author{
A. Phasouliotis D.K.C. So W. Prawatmuang \\ School of Electrical and Electronic Engineering, The University of Manchester, Oxford Road, Manchester, UK \\ E-mail: antonis.phasouliotis@manchester.ac.uk
}

\begin{abstract}
Energy efficiency has become increasingly important in wireless communications nowadays. Saving energy will not only reduce operating cost but also reduces greenhouse gas emissions, which is important for combating climate change. The authors propose energy-efficient user grouping algorithms to provide power minimisation of grouped multi-carrier code division multiple access (MC-CDMA) and space-time block coding MC-CDMA systems in a cellular environment. Depending on the channel fading conditions, power control is utilised to minimise the total transmitted power under a bit error rate constraint. When the allocation is performed without a fair data rate requirement, the authors provide the optimal solution to the minimisation problem. However, when some fairness is considered, the optimal solution requires high computational complexity. Thus, the authors solve the problem by proposing two suboptimal algorithms. Simulation results illustrate a significantly reduced power consumption in comparison with other techniques.
\end{abstract}

\section{Introduction}

With the increasing demand in wireless services and high data rate applications, reducing energy consumption is an important target for future wireless communication systems in order to sustain energy-efficient networks. Saving energy will not only reduce operating cost but also reduces $\mathrm{CO}_{2}$ emissions, which is important for combating climate change. For this reason, reducing the power consumption to a minimum level is vital for the future wireless systems.

Multi-carrier code division multiple access (MC-CDMA) [1-3] permits multiple users to access the wireless channel simultaneously by modulating and spreading their input data signals across the frequency domain using different spreading sequences. MC-CDMA combines the robustness to multipath fading by orthogonal frequency division multiplexing (OFDM), with the enhanced frequency diversity that can be achieved by code division multiple access (CDMA). To further exploit both spatial diversity and channel coding in multi-input multi-output systems and with low complexity receiver implementation, space-time block coding (STBC) has been combined with MC-CDMA systems in the past years [4-7]. Furthermore, to improve the quality-of-service, resource allocation has been investigated for MC-CDMA systems. To the best of our knowledge, existing works so far on resource allocation are mainly focused in maximising the total capacity in MC-CDMA [8-11].

Energy-efficient resource allocation has been investigated for OFDMA systems in $[12,13]$. However, to the best of our knowledge not a lot of work has been done for energyefficient MC-CDMA systems. Especially when using resource allocation to minimise the total transmitted power in MC-CDMA, the energy efficiency of the system can be improved significantly. In [14], rate, sub-carrier and power allocations are proposed for a multi-rate uplink MC-CDMA system with linear minimum mean square error multiuser detection to minimise total transmitted power based on different users' data rate and bit error rate (BER) requirements. Also in [11], a joint precoding and power allocation method is proposed for downlink multi-input single-output MC-CDMA systems, which minimises the total transmitted power of the system under a constraint on the signal-to-interference and noise ratio (SINR) of each user.

This work focuses on resource allocation for grouped MC-CDMA systems. In [15], Huang and Niu proposed a suboptimal user grouping and subcarrier allocation algorithm to maximise the total system throughput in grouped MC-CDMA. Also, the optimal power allocation for maximising the overall capacity in grouped MC-CDMA is given in [16]. The idea of user and subcarrier grouping [17] allows different users in a group to share the same set of subcarriers while using their distinct spreading codes. In this way multiuser interference in each group is small and does not affect users in the rest of the groups. Hence multiuser detectors for different groups are practically feasible. Although some research has been done for the maximisation of the total capacity in MC-CDMA, to the best of our knowledge, resource allocation with user grouping has not been considered to minimise the total transmitted power in grouped MC-CDMA systems.

In this paper, we study the minimisation of the total transmitted power in downlink MC-CDMA under a BER 
constraint for each user by performing power control according to an efficient user grouping algorithm. It should be noted that perfect channel information of the users are assumed both at the transmitter and the receiver. When there is no fairness requirement on the data rate, we present the optimal allocation algorithm for the minimisation problem. However, when fairness is considered, the optimal solution requires high computational complexity and is not favourable for practical applications. In this case we propose two complexity reduced suboptimal allocation algorithms. The energy efficiency of the proposed algorithms is evaluated in terms of the energy consumption gain (ECG) and the energy consumption ratio (ECR) [18] in a macrocell environment where path loss and shadowing are also considered.

The rest of this paper is organised as follows. Section 2 introduces the system model for the grouped MC-CDMA and STBC MC-CDMA systems. In Section 3, the formulation of the optimisation problems are presented. Section 4 provides the user grouping and power allocation algorithms. Simulation results are illustrated in Section 5. Finally, Section 6 concludes and summarises the paper.

Notations used in this paper are as follows. Bold lower and upper case letters denote vectors and matrices, respectively, whereas scalars are indicated by italic lower case fonts. Superscripts $(\cdot)^{\mathrm{T}},(\cdot)^{\mathrm{H}}$ and $(\cdot)^{-1}$ denote transpose, Hermitian transpose and matrix inversion, respectively. Matrix $I_{N}$ signifies an identity matrix of size $N \times N$ and $\|\cdot\|_{F}^{2}$ denotes the Frobenious norm.

\section{System models}

\subsection{Grouped MC-CDMA signal model}

Consider a downlink MC-CDMA system with $N_{u}$ users and Rayleigh fading channel. The channel is divided into $N_{S}$ subcarriers which are further grouped consecutively into $G$ groups. Each group uses a set of Walsh-Hadamard spreading sequences, with length $K=N_{s} / G$. Assuming multicode transmission is not used, each user in a group can only transmit one symbol over $K$ subcarriers. Thus the maximum number of users per group $U$ equals to the spreading code length $K$. The total number of allocatable resource units is therefore $G \times K$ which equals to $N_{s}$. The same set of spreading sequence is used in all other groups. Hence in any one group the signature sequence of user $u$ in that group $(u=1,2, \ldots, U)$ is expressed as $c_{u}=\left[\begin{array}{llll}c_{u, 1} & c_{u, 2} & \cdots & c_{u, K}\end{array}\right]^{\mathrm{T}^{\vec{T}} \in C^{K \times 1}}$ in which $C$ refers to the spreading code chip alphabet. The received signal model at the $u$ th user terminal of the gth group $(g=1,2, \ldots, G)$ can be characterised as

$$
\boldsymbol{r}_{u}^{g}=\boldsymbol{H}_{u}^{g} \boldsymbol{C} \boldsymbol{P}^{g} \boldsymbol{P} \boldsymbol{L}^{g} \boldsymbol{d}^{g}+\boldsymbol{v}_{u}^{g}
$$

where the users' symbol vector and additive white Gaussian noise (AWGN) vector with power $N_{0}$ are represented, respectively, by $\boldsymbol{d}^{g}=\left[\begin{array}{llll}d_{1}^{g} & d_{2}^{g} & \cdots & d_{U}^{g}\end{array}\right]^{\mathrm{T}} \in \mathbb{C}^{U \times 1}$ and $\boldsymbol{v}_{u}^{g}=\left[\begin{array}{cccc}v_{u, 1}^{g} & v_{u, 2}^{g} & \cdots & v_{u, K}^{g}\end{array}\right]^{\mathrm{T}} \in \mathbb{C}^{K \times 1}$. The transmit power matrix is represented by $\boldsymbol{P}^{g}=\operatorname{diag}\left(\sqrt{\rho_{1}^{g}}, \sqrt{\rho_{2}^{g}}, \ldots, \sqrt{\rho_{U}^{g}}\right) \in$ $\mathbb{R}^{U \times U}$ and the spreading code matrix is given by $\boldsymbol{C}=\left[\begin{array}{llll}\boldsymbol{c}_{1} & \boldsymbol{c}_{2} & \cdots & \boldsymbol{c}_{U}\end{array}\right] \in \mathbb{C}^{K \times U}$. The channel path loss and shadowing is represented by $\boldsymbol{P L}^{g}=\operatorname{diag}\left(\sqrt{1 / l_{1}^{g}}\right.$, $\sqrt{1 / l_{2}^{g}}, \ldots, \sqrt{\left.1 / l_{U}^{g}\right)} \in \mathbb{R}^{U \times U}$, where $l_{u}^{g}$ denotes the individual path loss and shadowing component for the $u$ th user in the gth group. The channel response matrix at the $u$ th user terminal of the gth group is denoted as $\boldsymbol{H}_{u}^{g}=$ $\operatorname{diag}\left(h_{u, 1}^{g}, h_{u, 2}^{g}, \ldots, h_{u, K}^{g}\right) \in \mathbb{C}^{K \times K}$ where $h_{u, k}^{g}$ represents the channel gain of the $u$ th user in the gth group at the $k$ th subcarrier $(k=1,2, \ldots, K)$. The received signal vector can be symbolised as

$$
\boldsymbol{r}_{u}^{g}=\left[\begin{array}{llll}
r_{u, 1}^{g} & r_{u, 2}^{g} & \cdots & r_{u, K}^{g}
\end{array}\right]^{\mathrm{T}}
$$

in which $r_{u, k}^{g}$ is the received signal of the $u$ th user in the gth group at the $k$ th subcarrier and it can be expressed as

$$
r_{u, k}^{g}=h_{u, k}^{g} \sum_{u^{\prime}=1}^{U} c_{u^{\prime}, k} \sqrt{\frac{\rho_{u^{\prime}}^{g}}{l_{u^{\prime}}^{g}}} d_{u^{\prime}}^{g}+v_{u, k}^{g}
$$

When the signal in (3) passes through a linear zero forcing (ZF) filter, the estimates of the $u$ th user in the gth group at the $k$ th subcarrier can be represented as

$y_{u, k}^{g}=\left(h_{u, k}^{g}\right)^{-1} r_{u, k}^{g}=c_{u, k} \sqrt{\frac{\rho_{u}^{g}}{l_{u}^{g}}} d_{u}^{g}+\sum_{u^{\prime}=1, u^{\prime} \neq u}^{U} c_{u^{\prime}, k} \sqrt{\frac{\rho_{u^{\prime}}^{g}}{l_{u^{\prime}}^{g}}} d_{u^{\prime}}^{g}+\tilde{v}_{u, k}^{g}$

where $\tilde{v}_{u, k}^{g}=\left(h_{u, k}^{g}\right)^{-1} v_{u, k}^{g}$. The $u$ th user's symbol decision statistic is obtained when the chip estimates in (4) are despread by the corresponding spreading sequence, given by

$$
z_{u}^{g}=\sum_{k=1}^{K} c_{u, k} y_{u, k}^{g}=\sqrt{\frac{\rho_{u}^{g}}{l_{u}^{g}}} d_{u}^{g}+\sum_{k=1}^{K} c_{u, k} \tilde{v}_{u, k}^{g}
$$

According to (5), it is obvious that multiple access interference (MAI) from other users in the group is cancelled by despreading. Thus power allocation for the $u$ th user can be performed by requiring only the $u$ th user's power information $\rho_{u}^{g}$. From (5), the received SINR for the $u$ th user of the gth group is calculated by

$$
\begin{aligned}
\operatorname{SINR}_{u}^{g} & =\frac{\rho_{u}^{g} E_{d}}{l_{u}^{g} \sum_{k=1}^{K} E_{h}\left[c_{u, k}\left(h_{u, k}^{g}\right)^{-1} v_{u, k}^{g} v_{u, k}^{g^{*}}\left(h_{u, k}^{g}\right)^{-1^{*}} c_{u, k}^{*}\right]} \\
& =\frac{K \rho_{u}^{g} E_{d}}{l_{u}^{g} N_{0} \sum_{k=1}^{K}\left[\left(h_{u, k}^{g}\right)^{-1}\left(h_{u, k}^{g}\right)^{-1^{*}}\right]}
\end{aligned}
$$

where $E_{h}[\cdot]$ and $(\cdot)^{*}$ refer to the conditional expectation on the channel gain $h$ and the complex conjugate operators, respectively, and $E_{d}$ refers to the symbol energy. Hence if there is a certain target SINR, denoted as $\gamma_{u}^{g}$, the transmitted power for the $u$ th user of the gth group is expressed as

$$
\rho_{u}^{g}=\frac{\gamma_{u}^{g} l_{u}^{g} N_{0}}{E_{d} K} \sum_{k=1}^{K}\left[\left(h_{u, k}^{g}\right)^{-1}\left(h_{u, k}^{g}\right)^{-1^{*}}\right]
$$

\subsection{Grouped STBC MC-CDMA signal model}

The signal model for the STBC MC-CDMA system is based on Alamouti's STBC with $N_{t}=2$ transmit and $N_{r}$ receive antennas. The system consists of $N_{u}$ users and each user experiences a Rayleigh fading channel. The available bandwidth is divided into groups in the same way as in the analysis for the MC-CDMA system. Each group uses the 
same set of spreading sequences as above. Furthermore, no multicode transmission is assumed, and hence each user in a group can only transmit one symbol over $K$ subcarriers. Thus, the maximum number of users per group $U$ equals to the spreading code length $K$. The total number of allocatable resource units is therefore $G \times K$ which equals to $N_{s}$.

During the Alamouti's transmission scheme, the $u$ th user transmits two symbols $d_{u}^{0}$ and $d_{u}^{1}$ over two consecutive transmissions. During the first transmission, $d_{u}^{0}$ and $d_{u}^{1}$ are transmitted simultaneously at time $t$ from the two transmit antennas. During the second transmission, $-d_{u}^{1 *}$ and $d_{u}^{0 *}$ are transmitted at time $t+T_{d}$ from the two transmit antennas, where $T_{d}$ denotes the OFDM symbol duration. The received signal model at the $u$ th user terminal of the gth group $(g=1,2, \ldots, G)$ for the $n_{r}$ th antenna $\left(n_{r}=1,2, \ldots, N_{r}\right)$ can be characterised by

$$
\begin{aligned}
\underbrace{\left[\begin{array}{c}
\boldsymbol{r}_{u, g}^{n_{r}}(1) \\
\boldsymbol{r}_{u, g}^{n_{r} H}(2)
\end{array}\right]}_{\boldsymbol{R}_{u, g}^{n_{r}}}= & \underbrace{\left[\begin{array}{cc}
\boldsymbol{H}_{u, g}^{1, n_{r}} & \boldsymbol{H}_{u, g}^{2, n_{r}} \\
\boldsymbol{H}_{u, g}^{2, n_{r} *} & -\boldsymbol{H}_{u, g}^{1, n_{r} *}
\end{array}\right]}_{\mathcal{H}_{u, g}^{n_{r}}} \underbrace{\left[\begin{array}{cc}
\boldsymbol{C} & 0 \\
0 & \boldsymbol{C}
\end{array}\right]}_{\mathcal{C}} \underbrace{\left[\begin{array}{cc}
\boldsymbol{\rho}_{g} & 0 \\
0 & \boldsymbol{\rho}_{g}
\end{array}\right]}_{\boldsymbol{P}_{g}} \\
& \times \underbrace{\left[\begin{array}{cc}
\boldsymbol{l}_{g} & 0 \\
0 & \boldsymbol{l}_{g}
\end{array}\right]}_{\boldsymbol{P} \boldsymbol{L}_{g}} \underbrace{\left[\begin{array}{c}
\boldsymbol{d}_{g}^{0} \\
\boldsymbol{d}_{g}^{1}
\end{array}\right]}_{\boldsymbol{D}_{g}}+\underbrace{\left[\begin{array}{c}
\boldsymbol{v}_{u, g}^{n_{r}}(1) \\
\boldsymbol{v}_{u, g}^{n_{r} H}(2)
\end{array}\right]}_{\boldsymbol{V}_{u, g}^{n_{r}}}
\end{aligned}
$$

where $\alpha^{\mathrm{H}}$ denotes the Hermitian transpose of $\alpha$. The received signal vector after the $i$ th transmission is expressed as $\boldsymbol{r}_{u, g}^{n_{r}}(i)=\left[\begin{array}{lll}r_{u, g, 1}^{n_{r}}(i) & \cdots & r_{u, g, K}^{n_{r}}(i)\end{array}\right]^{\mathrm{T}} \in \mathbb{C}^{K \times 1}$ in which $r_{u, g, k}^{n_{r}}(i)$ symbolises the received signal of the $u$ th user in the gth group at the $k$ th subcarrier $(k=1,2, \ldots, K)$ for the $n_{r}$ th antenna which is received after the ith transmission. The channel response matrix of the $u$ th user terminal in the gth group at the $n_{t}$ th transmit and $n_{r}$ th receive antenna is denoted as $H_{u, g}^{n_{t}, n_{r}}=\operatorname{diag}\left(h_{u, g, 1}^{n_{t}, n_{r}}, h_{u, g, 2}^{n_{t}, n_{r}}, \ldots, h_{u, g, K}^{n_{t}, n_{r}}\right) \in \mathbb{C}^{K \times K}$ where $h_{u, g}^{n_{t}, n_{r}}$ represents the channel gain of the $u$ th user in the gth group for the $k$ th subcarrier at the $n_{t}$ th transmit and $n_{r}$ th receive antennas. The users' symbol vector is represented by $\boldsymbol{d}_{g}^{j}=\left[\begin{array}{lll}d_{1, g}^{j} & \cdots & d_{U, g}^{j}\end{array}\right]^{\mathrm{T}} \in \mathbb{C}^{U \times 1}$ and the AWGN vector with power $N_{0}$ is represented by $\boldsymbol{v}_{u, g}^{n_{r}}(i)=$ $\left[\begin{array}{lll}v_{u, g, 1}^{n_{r}}(i) & \cdots & v_{u, g, K}^{n_{r}}(i)\end{array}\right]^{\mathrm{T}} \in \mathbb{C}^{K \times 1}$. The transmit power and path loss vectors are represented, respectively, by $\boldsymbol{\rho}_{g}=\operatorname{diag}\left[\begin{array}{lll}\sqrt{\rho_{1, g} / 2} & \cdots & \sqrt{\rho_{U, g} / 2}\end{array}\right] \in \mathbb{R}^{U \times U} \quad$ and $\quad \boldsymbol{l}_{g}=$ $\operatorname{diag}\left[\begin{array}{lll}\sqrt{1 / l_{1, g}} & \cdots & \sqrt{1 / l_{U, g}}\end{array}\right] \in \mathbb{R}^{U \times U}$, and the spreading code matrix is given by $\boldsymbol{C}=\left[\begin{array}{lll}\boldsymbol{c}_{1} & \cdots & \boldsymbol{c}_{U}\end{array}\right] \in \mathbb{C}^{K \times U}$. Considering the signal in (8) passing through a linear $\mathrm{ZF}$ filter, the output signal can be expressed by

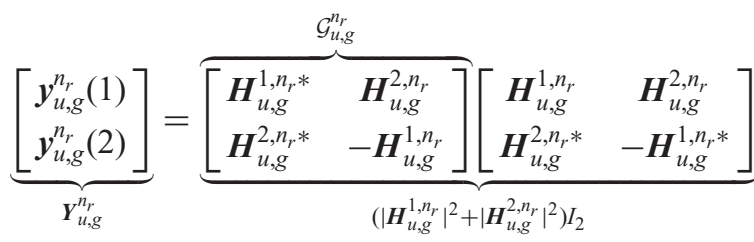

$$
\begin{aligned}
& \times\left[\begin{array}{ll}
\boldsymbol{C} & 0 \\
0 & \boldsymbol{C}
\end{array}\right]\left[\begin{array}{cc}
\boldsymbol{\rho}_{g} & 0 \\
0 & \boldsymbol{\rho}_{g}
\end{array}\right]\left[\begin{array}{cc}
\boldsymbol{l}_{g} & 0 \\
0 & \boldsymbol{l}_{g}
\end{array}\right]\left[\begin{array}{c}
\boldsymbol{d}_{g}^{0} \\
\boldsymbol{d}_{g}^{1}
\end{array}\right] \\
& +\overbrace{\left[\begin{array}{cc}
\boldsymbol{H}_{u, g}^{1, n_{r} *} & \boldsymbol{H}_{u, g}^{2, n_{r}} \\
\boldsymbol{H}_{u, g}^{2, n_{r} *} & -\boldsymbol{H}_{u, g}^{1, n_{r}}
\end{array}\right]}^{\mathcal{G}_{u, g}^{n_{r}}}\left[\begin{array}{c}
\boldsymbol{v}_{u, g}^{n_{r}}(1) \\
\boldsymbol{v}_{u, g}^{n_{r} H}(2)
\end{array}\right]
\end{aligned}
$$

where the equalised signal of the $u$ th user in the gth group at the $n_{r}$ th antenna is given by $\boldsymbol{y}_{u, g}^{n_{r}}(i)=$ $\left[\begin{array}{lll}y_{u, g, 1}^{n_{r}}(i) & \cdots & y_{u, g, K}^{n_{r}}(i)\end{array}\right]^{\mathrm{T}} \in \mathbb{C}^{K \times 1}$. The $u$ th user's symbols decision statistic is obtained when the two successive equalised signals in (9) are space-time combined and despread by the corresponding spreading sequence. This is represented by

$$
\begin{aligned}
\boldsymbol{z}_{u, g}= & \left(\boldsymbol{I}_{2} \otimes \boldsymbol{c}_{u}^{\mathrm{T}}\right) \sum_{n_{r}=1}^{N_{r}} \boldsymbol{Y}_{u, g}^{n_{r}} \\
= & \sqrt{\frac{\rho_{u, g}}{2 l_{u, g}}}\left[\sum_{k=1}^{K} \sum_{n_{r}=1}^{N_{r}}\left|h_{u, g, k}^{1, n_{r}}\right|^{2}+\left|h_{u, g, k}^{2, n_{r}}\right|^{2}\right]\left[\begin{array}{c}
d_{u, g}^{0} \\
d_{u, g}^{1}
\end{array}\right] \\
& +\left(\boldsymbol{I}_{2} \otimes \boldsymbol{c}_{u}^{\mathrm{T}}\right) \sum_{n_{r}=1}^{N_{r}} \mathcal{G}_{u, g}^{n_{r}} \boldsymbol{V}_{u, g}^{n_{r}}
\end{aligned}
$$

where $\quad \boldsymbol{z}_{u, k}=\left[z_{u, k}(1) \quad z_{u, k}(2)\right]^{\mathrm{T}} \in \mathbb{C}^{2 \times 1} \quad$ and $\otimes$ is the Kronecker product. According to (10), MAI from other users in the group is cancelled by despreading. Thus power allocation for the $u$ th user can be performed by requiring only the $u$ th user's power information $\rho_{u, g}$. From (10), the received SINR for the $u$ th user of the gth group can be calculated by

$$
\begin{aligned}
\operatorname{SINR}_{u}^{g}= & \frac{\frac{\rho_{u, g}}{2 l_{u, g}} E_{d}\left[\sum_{k=1}^{K} \sum_{n_{r}=1}^{N_{r}}\left|h_{u, g, k}^{1, n_{r}}\right|^{2}+\left|h_{u, g, k}^{2, n_{r}}\right|^{2}\right]^{2}}{\frac{N_{0}}{K} \sum_{k=1}^{K} \sum_{n_{r}=1}^{N_{r}}\left|h_{u, g, k}^{1, n_{r}}\right|^{2}+\left|h_{u, g, k}^{2, n_{r}}\right|^{2}} \\
= & \frac{\rho_{u, g} K E_{d}}{2 l_{u, g} N_{0}} \sum_{k=1}^{K} \sum_{n_{r}=1}^{N_{r}}\left|h_{u, g, k}^{1, n_{r}}\right|^{2}+\left|h_{u, g, k}^{2, n_{r}}\right|^{2} \\
= & \frac{\rho_{u, g} K E_{d}}{2 l_{u, g} N_{0}} \sum_{k=1}^{K} \operatorname{tr}\left(\overline{\boldsymbol{H}}_{u, g, k}^{H} \overline{\boldsymbol{H}}_{u, g, k}\right)
\end{aligned}
$$

where

$$
\overline{\boldsymbol{H}}_{u, g, k}=\left[\begin{array}{cc}
h_{u, g, k}^{1,1} & h_{u, g, k}^{2,1} \\
h_{u, g, k}^{1,2} & h_{u, g, k}^{2,2} \\
\vdots & \vdots \\
h_{u, g, k}^{1, n_{r}} & h_{u, g, k}^{2, n_{r}}
\end{array}\right]
$$

and $\operatorname{tr}(\boldsymbol{A})$ denotes the trace of matrix $\boldsymbol{A}$. Hence if there is a certain target SINR, denoted as $\gamma_{u}^{g}$, the transmitted power for the $u$ th user of the gth group is expressed as

$$
\rho_{u, g}=\frac{2 \gamma_{u}^{g} l_{u, g} N_{0}}{K E_{d} \sum_{k=1}^{K} \operatorname{tr}\left(\overline{\boldsymbol{H}}_{u, g, k}^{H} \overline{\boldsymbol{H}}_{u, g, k}\right)}
$$

\section{Problem formulation}

In this section we consider the minimisation of MC-CDMA and STBC MC-CDMA system's total transmitted power under a BER constraint by performing user grouping and power control. The minimisation problems for both systems are formulated with and without fairness criteria. Fairness is applied to ensure some bandwidth is allocated to each user. 
The total transmitted power of the MC-CDMA system can be expressed as

$$
P_{T}=\sum_{g=1}^{G} \sum_{u=1}^{U} \rho_{u}^{g}=\sum_{g=1}^{G} \sum_{u=1}^{U} \frac{\gamma_{u}^{g} N_{0}}{E_{d} K} \omega_{u}^{g}
$$

where $\omega_{u}^{g}$ represents a channel related factor of the $u$ th user in the gth group given by

$$
\omega_{u}^{g}=l_{u}^{g} \sum_{k=1}^{K}\left[\left(h_{u, k}^{g}\right)^{-1}\left(h_{u, k}^{g}\right)^{-1^{*}}\right]
$$

To group users for power minimisation, the following channel related factor for all system users $\left(n_{u}=1, \ldots, N_{u}\right)$ has to be considered

$$
\bar{\omega}_{n_{u}}^{g}=l_{n_{u}}^{g} \sum_{k=1}^{K}\left[\left(h_{n_{u}, k}^{g}\right)^{-1}\left(h_{n_{u}, k}^{g}\right)^{-1^{*}}\right]
$$

Using the probability of error for quadrature phase shift keying modulation in [19], the target SINR can be expressed with respect to a target BER as

$$
\gamma_{u}^{g}=1 / 2\left[Q^{-1}\left(\mathrm{BER}_{u}^{g} / 2\right)\right]^{2}
$$

Hence the optimisation problem without any fairness criteria can be formulated mathematically as

$$
\begin{gathered}
\min _{\xi_{\xi_{u}}^{g}} \sum_{g=1}^{G} \sum_{n_{u}=1}^{N_{u}} \frac{N_{0}\left[Q^{-1}\left(\mathrm{BER}_{u}^{g} / 2\right)\right]^{2}}{2 E_{d} K} \bar{\omega}_{n_{u}}^{g} \xi_{n_{u}}^{g} \\
\text { s.t. } \quad \mathrm{BER}_{u}^{g} \leq \overline{\mathrm{BER}} \\
\sum_{n_{u}=1}^{N_{u}} \xi_{n_{u}}^{g}=K, \quad \forall g=1,2, \ldots, G
\end{gathered}
$$

in which $\xi_{n_{u}}^{g} \in\{0,1\}$ is an indicator function with ' 1 ', denoting user $n_{u}$ being allocated to group $g$ and ' 0 ' otherwise. Constraint (19) requires that the instantaneous BER of the $u$ th user of the gth group is equal to or smaller than the minimum BER value $\overline{\mathrm{BER}}$, whereas constraint (20) ensures that each group consists of $K$ users.

The minimisation for STBC MC-CDMA is formulated in a similar way as for MC-CDMA. By performing user grouping and power control, we consider the minimisation of the system's total transmitted power under a BER constraint. The total transmitted power of the system can be characterised as

$$
P_{T}=\sum_{g=1}^{G} \sum_{u=1}^{U} \rho_{u, g}=\sum_{g=1}^{G} \sum_{u=1}^{U} \frac{2 \gamma_{u}^{g} N_{0}}{K E_{d}} \omega_{u}^{g}
$$

where $\omega_{u}^{g}$ is denoted by

$$
\omega_{u}^{g}=\frac{l_{u, g}}{\sum_{k=1}^{K} \operatorname{tr}\left(\overline{\boldsymbol{H}}_{u, g, k}^{H} \overline{\boldsymbol{H}}_{u, g, k}\right)}
$$

The channel related factor for all system users can be expressed as

$$
\overline{\boldsymbol{\omega}}_{n_{u}}^{g}=\frac{l_{n_{u}, g}}{\sum_{k=1}^{K} \operatorname{tr}\left(\overline{\boldsymbol{H}}_{n_{u}, g, k}^{H} \overline{\boldsymbol{H}}_{n_{u}, g, k}\right)}
$$

Similar to the MC-CDMA analysis, the optimisation problem without any fairness criteria can be formulated mathematically as

$$
\begin{gathered}
\min _{\xi_{n_{u}}} \sum_{g=1}^{G} \sum_{n_{u}=1}^{N_{u}} \frac{N_{0}\left[Q^{-1}\left(\mathrm{BER}_{u}^{g} / 2\right)\right]^{2}}{K E_{d}} \bar{\omega}_{n_{u}}^{g} \xi_{n_{u}}^{g} \\
\text { s.t. } \quad \mathrm{BER}_{u}^{g} \leq \overline{\mathrm{BER}} \\
\sum_{n_{u}=1}^{N_{u}} \xi_{n_{u}}^{g}=K, \quad \forall g=1,2, \ldots, G
\end{gathered}
$$

in which $\xi_{n_{u}}^{g} \in\{0,1\}$ and the two constraints are the same as in the problem formulation for MC-CDMA.

\section{Problem optimisation}

Having formulated the problems for MC-CDMA and STBC MC-CDMA in the previous section, we propose several power-minimising user grouping algorithms in this section. First, the optimal algorithm based on Greedy approach is proposed when there is no fairness criterion in the data rate. However, when fairness is applied, the optimal solution requires high computational complexity and is not favourable for practical applications. Therefore we propose two suboptimal allocation algorithms. The performances of these algorithms are evaluated in the next section.

\subsection{Greedy allocation}

The optimal solution to the minimisation problem with no fairness criterion is first presented. Since we assume that users can be allocated to more than one group of subcarriers, the optimum solution to the minimisation problem in (18) or (24) would be to allocate users with the smallest $\bar{\omega}_{n_{u}}^{g}$ to the corresponding gth group. Hence the total transmitted power can be minimised if we only allow users with the largest channel gains to use the available subcarriers. The Greedy algorithm can be described as follows.

Step 1: Divide the available bandwidth into $G=N_{s} / K$ groups. Each group consists of $U=K$ available positions for user assignment.

Step 2: For all $G$ groups, calculate $\bar{\omega}_{n}^{g}$ for every $n u$ th user according to (16) for MC-CDMA and (23) for STBC MCCDMA.

Step 3: Allocate the $U$ users with the smallest $\bar{\omega}_{n_{u}}^{g}$ in the gth group.

Step 4: Repeat Step 3 until all groups are assigned with $U$ users.

Step 5: Calculate the total transmitted power according to (14) for MC-CDMA and (21) for STBC MC-CDMA by setting $\operatorname{BER}_{u}^{k}=\overline{\mathrm{BER}}$.

By selecting users with the lowest $\bar{\omega}_{n_{u}}^{g}$, it is evident from (14) and (21) that the total transmitted power for every group and hence for the system will be minimised. This satisfies the minimisation problem in in (18) and (24) with the constraints in (19), (20) and (25), (26) respectively. 


\subsection{Allocation with fairness criterion}

When there is no fairness requirement, users with low channel gains, that is, weaker users in the system, may not be allocated to any group and hence not be able to transmit their data. For this reason the fairness criterion is considered such that each user has to be assigned to a group at least once, that is, each user can transmit at least one symbol before the Greedy allocation is applied. In this way it is possible for all users to transmit their data. The fairness constraint can be imposed as

$$
\sum_{g=1}^{G} \xi_{n_{u}}^{g} \geq 1, \quad \forall n_{u}=1,2, \ldots, N_{u}
$$

The optimal solution to the minimisation problem with this additional fairness constraint is given by calculating the total transmitted power for all possible combinations of user allocations, and choosing the one that gives the minimum total power. However, this method requires very high computational complexity. Hence we propose a simple suboptimal algorithm that requires less complexity and we call this Fairness A algorithm.

Step 1: Follow Steps 1 and 2 in Greedy algorithm.

Step 2: Set up the list $\mathcal{L} \in\left\{1, \ldots, N_{u}\right\}$ that contains all the unallocated users.

Step 3: Allocate the user with the smallest $\bar{\omega}_{n,}^{g}$ from $\mathcal{L}$, in the gth group. Remove this allocated user from $\mathcal{L}$.

Step 4: Repeat Step 3 until each user is assigned in a group once, that is, until $\mathcal{L}$ becomes a null set. If a group has already been allocated with $U$ users, this group is filled and will not be allocated anymore users.

Step 5: If not all groups are assigned with $U$ users, follow Steps 3 and 4 of the Greedy algorithm.

Step 6: Calculate the total transmitted power as in Step 5 of the Greedy algorithm.

The Fairness A algorithm is a simple and low complexity solution. However, as will be shown in Section 5, it has poor performance when the number of users approaches the maximum supportable by the system, that is, $N_{u} \rightarrow G K$. This is because this algorithm first allocates users with large channel gains to the group containing their best subcarriers. When a group is filled, the remaining users will be allocated to other groups, even though their best subcarriers may lie in this group. Thus these remaining users could be allocated with poorer subcarriers, and this requires a larger transmission power. As the number of users increases, the groups will be filled more quickly and hence this situation occurs more often. Therefore the total system transmission power will be significantly increased.

To overcome this problem, we propose a second suboptimal algorithm (Fairness B) that considers the amount of channel variation over the entire bandwidth. Consider a scenario where two users have their best subcarriers in the same group, but there is only one resource unit left in that group. User 1 has a larger but relatively flat channel gain over the entire bandwidth, while User 2 has a smaller gain but with larger channel variation. The Fairness A algorithm will assign User 1 to this group due to its larger gain. User 2 will then be allocated to another group, which might have a much lower channel gain due to the large channel variation. On the contrary if User 2 was allocated to this group, User 1 will be allocated to another group having a similarly large channel gain due to its relatively flat channel response. Hence, both users will be allocated to good channels, and the total transmission power can be significantly reduced.

Therefore it is important to group users with large channel variation first, such that their best subcarriers are used. Users with flatter channel responses should be allocated later as the difference between the subcarriers is not significant. The proposed Fairness B algorithm measures channel variation by the standard deviation of the channel related factor $\bar{\omega}_{n_{u}}^{g}$ for each user, and is detailed below.

Step 1: Same as Steps 1 and 2 in Greedy algorithm.

Step 2: For each user, calculate the standard deviation of $\bar{\omega}_{n_{u}}^{g}$ over the $G$ groups. Rank the users in a descending order of this standard deviation.

Step 3: Follow the ranking order to allocate the next user to the group with its lowest $\bar{\omega}_{n_{u}}^{g}$. Remove this allocated user from the ranking list.

Step 4: Repeat Step 3 until each user is assigned one resource unit. If a group is filled, no more users can be assigned to that group.

Step 5: Same as Step 5 in Greedy algorithm.

\section{Simulation results and discussions}

In this section, the performances of the proposed algorithms are evaluated through Monte-Carlo simulations. Consider a single-input single-output MC-CDMA system and a $2 \times 1$ and $2 \times 2$ antenna configuration for the STBC MC-CDMA system in a single macrocell environment. The users are uniformly distributed over a $2 \mathrm{~km} \times 2 \mathrm{~km}$ square with the base station located at the centre of the cell. The detailed cell parameters are given in Table 1. Each spreading sequence in the system is assumed to comprise eight chips $(K=8)$ and Walsh-Hadamard spreading sequences are used for each user. The finite Fourier transform size is considered to be the same as the number of subcarriers and is set to be $64\left(N_{s}=64\right)$. Thus the available bandwidth is divided into eight groups $(G=8)$, each consisting of eight users $(U=8)$ and eight subcarriers. The channel is assumed to be a typical urban area propagation model specified in [20], with six taps and the parameters are listed in Table 2. It is also assumed that the maximum delay spread is shorter than the duration of the cyclic prefix. Hence, inter-state interference is avoided and each chip

Table 1 System and cell parameters

Cell parameters structure

Tx antenna height

Rx antenna height

Tx antenna gain

$\mathrm{Rx}$ antenna gain

carrier frequency

total bandwidth

noise spectral density

$\mathrm{Rx}$ noise figure

shadowing standard deviation
$2 \mathrm{~km} \times 2 \mathrm{~km}$ square $35 \mathrm{~m}$

$1.8 \mathrm{~m}$

$13 \mathrm{dBi}$

$0 \mathrm{dBi}$

$1.9 \mathrm{GHz}$

$5 \mathrm{MHz}$

$-174 \mathrm{dBm} / \mathrm{Hz}$

$5 \mathrm{~dB}$

Table 2 Relative powers of delay profile [20]

\begin{tabular}{lcccccc}
\hline delay, $\mu \mathrm{s}$ & 0.0 & 0.2 & 0.6 & 1.6 & 2.4 & 5.0 \\
relative power, $\mathrm{dB}$ & -3.0 & 0.0 & -2.0 & -6.0 & -8.0 & -10.0 \\
\hline
\end{tabular}


experiences flat fading. Furthermore, the channel path loss is calculated using the COST231 metropolitan city model [21].

The aim in this work is to show the energy efficiency of the proposed schemes. For this reason the ECR and the ECG are chosen to be the performance measures. ECR is defined as the ratio of the total consumed energy that is dissipated in the macrocell network over the maximum data throughput $\left(P_{M} /\right.$ $R_{T}$ ) and is measured in Joules per bit. This performance measure is important as it shows how much power should be invested in the system per information bit. ECG is the ratio between the total energy consumed by the baseline system divided by the energy consumed by the system under test $\left(P_{b} / P_{t}\right)$. We consider the power consumption model of a macrocell from [22] as $P_{M}=\alpha P_{T}+P_{\text {fixed }}$ where $P_{T}$ represents the total transmit power of the system. According to this model, $\alpha=3.8$ and denotes a scaling factor of the average radiated power due to amplifier and feeder losses as well as cooling of sites. Furthermore, $P_{\text {fixed }}=68.8 \mathrm{~W}$ and models fixed site power which is consumed independently of the average transmit power due to signal processing, battery backup, as well as site cooling. Another performance measure to illustrate power minimisation is chosen to be the total transmit power over the noise level $\left(P_{T} / N_{0}\right)$ in $\mathrm{dB}$.

Fig. 1 demonstrates the $P_{T} / N_{0}$ with respect to different number of users for our proposed algorithms and the random user grouping algorithm in MC-CDMA systems. For these results we assume a target BER value of $10^{-4}$ $\left(\overline{\mathrm{BER}}=10^{-4}\right)$.

The results show that Fairness $\mathrm{A}$ and $\mathrm{B}$ algorithms significantly outperform the random allocation algorithm. Further power reduction can be obtained when the Greedy algorithm is performed. Hence when there is no fairness requirement in the user selection process, more power can be saved since we only assign subcarriers to the stronger users. However, weaker users in the system will not be able to send their data. Furthermore, the results for Greedy algorithm depict that the total transmitted power decreases as the number of users increases. This is because with more users in the system, there will be higher multiuser diversity.

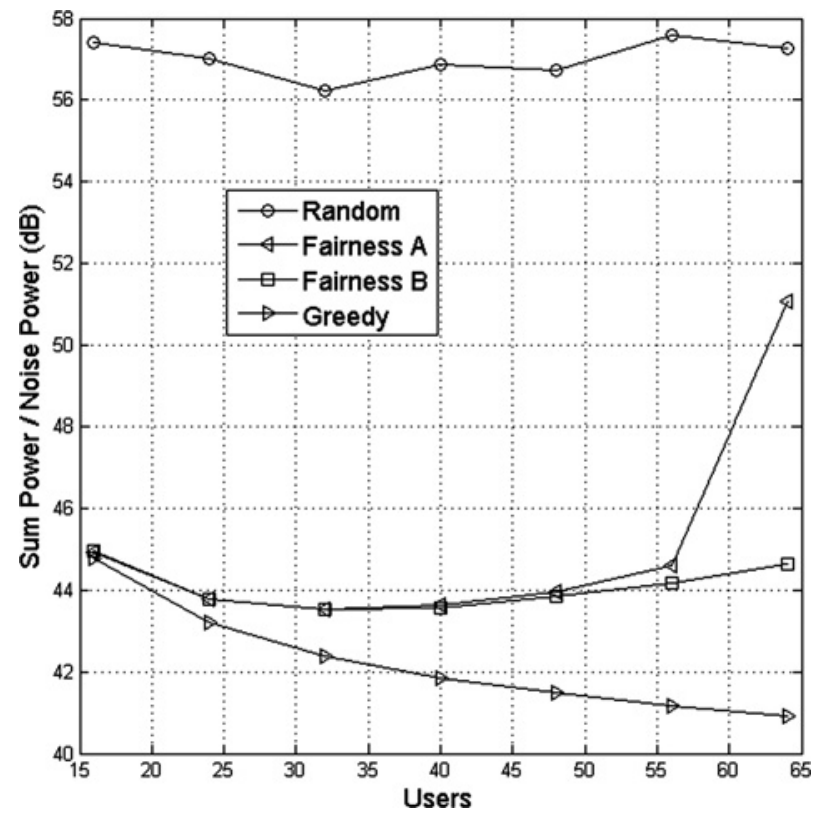

Fig. 1 Total system transmitted power for different number of users
Thus, it is more likely to assign users onto better subcarriers and hence the total power becomes smaller.

When the total number of users increases towards the total number of subcarriers, a significant increase in power consumption is observed for Fairness A algorithm. As explained in the previous section, the assignment of weaker users into groups with deep faded subcarriers increases the total power consumption. With the use of Fairness B algorithm, the increase in power is avoided. This is because Fairness B first allocates the users with higher standard deviation with respect to $\bar{\omega}_{n_{u}}^{g}$, that is, the users with larger channel variation. Hence these users are allocated to the group where they will require less power. Users with lower channel variation are subsequently assigned, which do not require significantly different transmission power. The results validated the proposed approach for Fairness B algorithm.

In Fig. 2, we evaluate the total transmitted power ratio for our proposed algorithms with respect to different target BER values. We also compare our results with the random user grouping algorithm. For these results we assume a full load system of $N_{u}=64$. It can be observed that at a target BER of $10^{-4}$, Fairness A can save $9 \mathrm{~dB}$ of transmission power in comparison to the random allocation. A further $4 \mathrm{~dB}$ of power reduction can be obtained when Fairness $B$ is utilised. Further observations show that if no fairness constraint is considered and the Greedy algorithm is used, a $4 \mathrm{~dB}$ decrement in power is achieved in comparison to the Fairness B algorithm. Thus, the Fairness B algorithm achieves good performance even though the fairness criterion is considered.

The proposed algorithms are also compared with respect to the ECR for different BER values and the results are presented in Fig. 3. The total transmitted power in the proposed algorithms is minimised under an equal target BER for each user. Thus, the instantaneous signal to noise ratio for each user is identical and hence the spectral efficiency for each user is the same. Considering a system with 64 users, observations at a BER of $10^{-4}$ illustrate that the Fairness A algorithm outperforms the random allocation. When Fairness B is considered, less energy per bit is required in comparison with Fairness A. Furthermore, more reduction in energy per bit is observed when the Greedy algorithm is used. Thus, the proposed algorithms are more energy efficient than the random allocation algorithm.

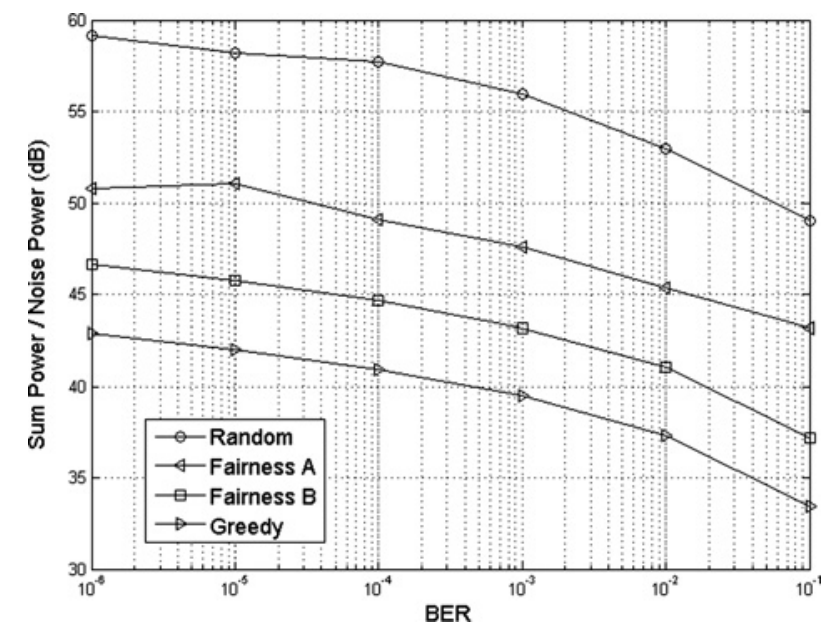

Fig. 2 Total system transmitted power against different target BERS 


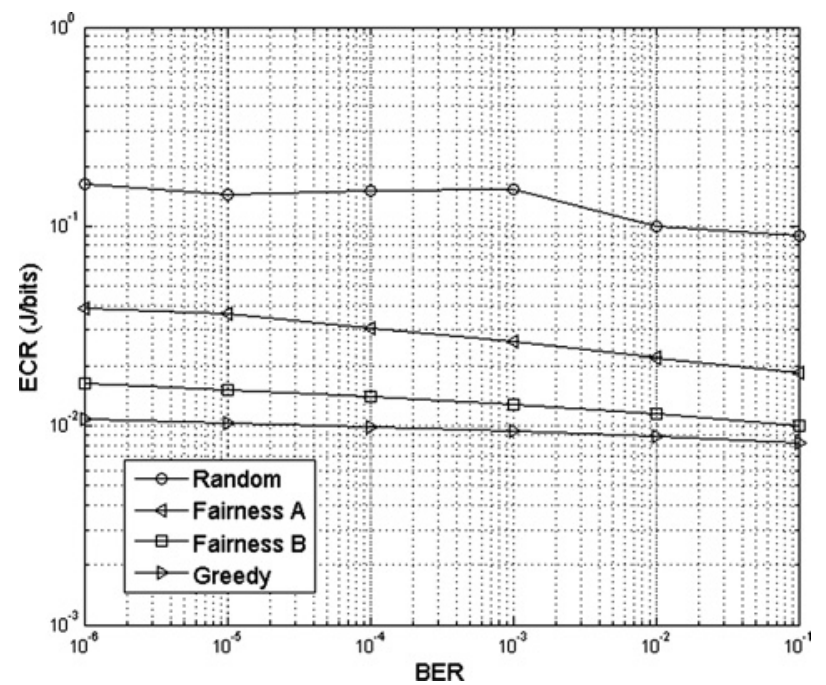

Fig. 3 ECR against different target BERs

Fig. 4 demonstrates the $P_{T} / N_{0}$ with respect to different number of users when the proposed algorithms and the random user grouping algorithm are applied in STBC MCCDMA systems. The results show that when multiple transmit and multiple receive antennas are applied in MCCDMA systems, the use of STBC can exploit spatial diversity to significantly reduce power consumption and increase energy efficiency in the system. Further observations show that Fairness $\mathrm{A}$ and $\mathrm{B}$ algorithms outperform the random allocation algorithm. Also, when the Greedy algorithm is performed, further power reduction can be obtained. Similar to the MC-CDMA system, the results for Greedy algorithm depict that the total transmitted power decreases as the number of users increases.

When the number of receive antennas increases to 2 , a decrease in power consumption is observed for all the algorithms. This is because with more receiving antennas there will be higher spatial diversity. Thus $\bar{\omega}_{n_{u}}^{g}$ in (23) gets smaller and the total power consumption is decreased.

In Fig. 5, we evaluate the total transmitted power ratio for our proposed algorithms with respect to different target BER

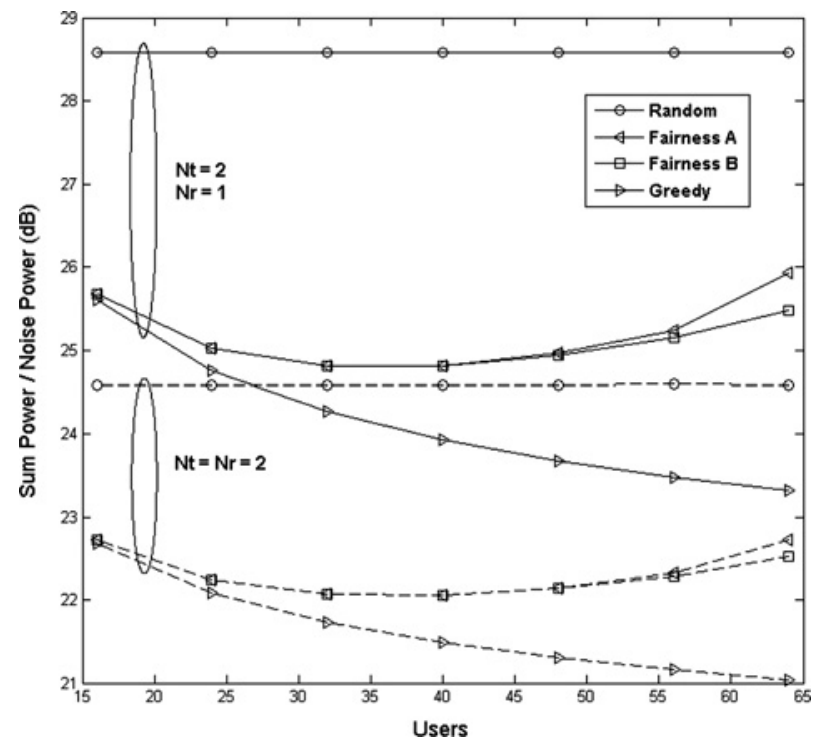

Fig. 4 Total system transmitted power for different number of users

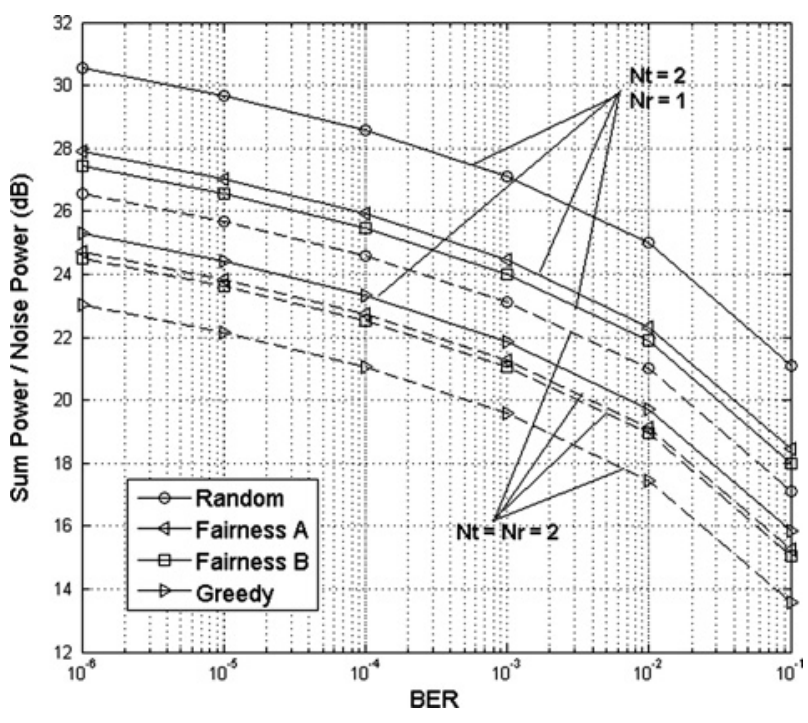

Fig. 5 Total system transmitted power against different target BERs

values. We also compare our results with the random user grouping algorithm. For these results we assume a full load system of $N_{u}=64$. It can be observed that at a target BER of $10^{-4}$ and when $N_{r}=1$, Fairness A can save $3 \mathrm{~dB}$ of transmission power in comparison to the random allocation. A further $0.5 \mathrm{~dB}$ of power reduction can be obtained when Fairness B is utilised. Further observations show that if no fairness constraint is considered and the Greedy algorithm is used, a $2 \mathrm{~dB}$ decrement in power is achieved in comparison to the Fairness B algorithm. It has to be noticed that as the number of receive antennas increases the power reduction between the different algorithms decreases.

To compare the energy saving of the proposed STBC MC-CDMA user grouping algorithms over the random allocation algorithm, we evaluate the ECG as shown in Fig. 6. It must be noted that the larger the ECG, the more energy efficient the system under test becomes. From the results, it is evident that Fairness A and B provide more energy efficiency than the random allocation. Further ECG is produced by the Greedy algorithm when the fairness criterion is not required.

When the number of receive antennas increases to 2, a decrease on EGC is produced for all the algorithms. This

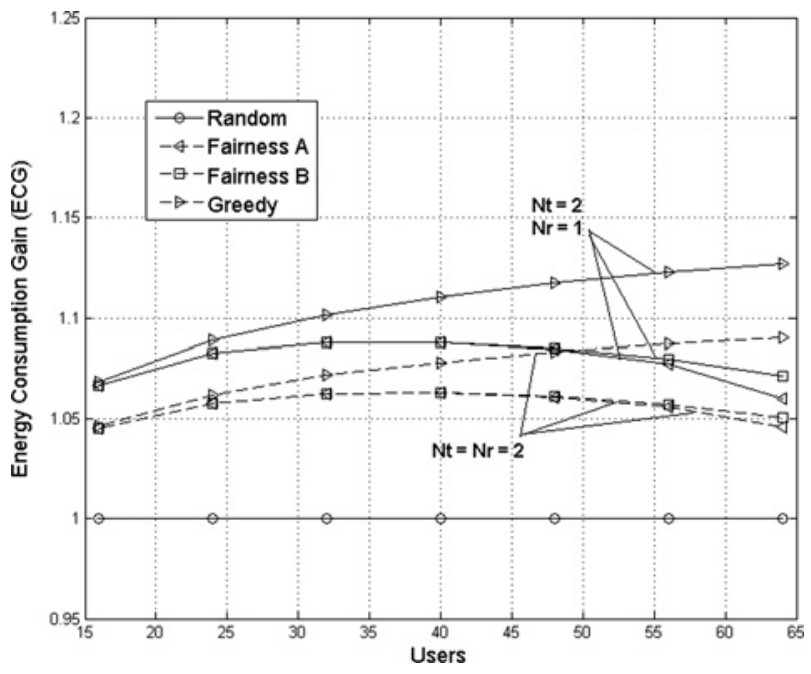

Fig. 6 ECG for different number of users 
happens because with more receiving antennas there is higher spatial diversity which allows the random allocation algorithm to save more power compared to the power saved by the other algorithms. For this reason the EGC of the other algorithms over the random allocation becomes lower.

\section{Conclusion}

In this paper, energy efficient and low complexity user grouping algorithms are proposed for grouped MC-CDMA and STBC MC-CDMA systems. The optimal solution for minimising the total transmitted power with respect to a target BER is proposed when no fairness requirement on the data rate is needed. When a fairness requirement is considered in the user grouping process, we propose two suboptimal algorithms for the optimisation problem. The first algorithm is a simple approach based on the subcarrier channel gains, while the second algorithm is based on the channel variation. Simulation results justify that our proposed algorithms provide a significant reduction in power consumption when compared with the random user allocation algorithm.

\section{Acknowledgment}

This paper was presented in part at the IEEE Vehicular Technology Conference (VTC'10), Ottawa, Canada, Sep 2010 and at the IEEE PIMRC'10, Istanbul, Turkey, September 2010 .

\section{References}

1 Yee, N., Linnartz, J.-P., Fettweis, G.: 'Multicarrier CDMA in indoor wireless radio networks'. Proc. IEEE PIMRC, September 1993, pp. 109-113

2 Fazel, K., Papke, L.: 'On the performance of convolutionally-coded CDMA/OFDM for mobile communication system'. Proc. IEEE PIMRC, September 1993, pp. 468-472

3 Chouly, A., Brajal, A., Jourdan, S.: 'Orthogonal multicarrier techniques applied to direct sequence spread spectrum CDMA systems'. Proc. IEEE GLOBECOM, November 1993, pp. 1723-1728

4 Nir, V.L., Helard, M., Gouable, R.L.: 'Space-time block coding applied to turbo coded multicarrier CDMA'. Proc. IEEE VTC Spring, April 2003, pp. 577-581
5 Yang, Z., Lu, B., Wang, X.: 'Bayesian Monte Carlo multiuser receiver for space-time coded multicarrier CDMA systems', IEEE J. Sel. Areas Commun., 2001, 19, (8), pp. 1625-1637

6 Zhihua, H., Dubey, V.: 'Performance analysis for downlink MCCDMA systems with space-time block codes in frequency-selective Rayleigh fading channels'. Proc. ICICS-PMC, December 2003, pp. $658-662$

7 Portier, F., Baudars, J., Helard, J.: 'Performance of STBC MC-CDMA systems over outdoor realistic MIMO channels'. Proc. IEEE VTC Fall, September 2004, pp. 2409-2413

8 Lo, E., Chan, P., Lau, V., et al:: 'Adaptive resource allocation and capacity comparison of downlink multiuser MIMO-MC-CDMA and MIMO-OFDMA', IEEE Trans. Wirel. Commun., 2007, 6, (2), pp. $1083-1093$

9 Costa, E., Haas, H., Schulz, E., Filippi, A.: 'Capacity optimisation in MC-CDMA systems', Eur. Trans. Telecommun., 2002, 13, (5), pp. $455-463$

10 Li, C., Wang, X.: 'Adaptive subchannel allocation in multiuser MC-CDMA systems'. Proc. IEEE GLOBECOM, December 2004 pp. 2503-2507

11 Benvenuto, N., Bisaglia, P., Boccardi, F.: 'Joint optimum linear precoding and power control strategies for downlink MC-CDMA systems', IEEE Trans. Commun., 2008, 56, (5), pp. 769-777

12 Akbari, A., Hoshyar, R., Tafazolli, R.: 'Energy-efficient resource allocation in wireless OFDMA systems'. Proc. IEEE PIMRC, September 2010, pp. 1731-1735

13 Miao, G.W., Himayat, N., Li, G.Y., Bormann, D.: 'Energy-efficient design in wireless OFDMA'. Proc. IEEE ICC, May 2008, pp. $3307-3312$

14 Fu, P., Chen, K.: 'Rate, sub-carrier, and power allocations for multicarrier CDMA with LMMSE multiuser detection', IEEE Trans. Wirel. Commun., 2007, 6, (5), pp. 1574-1580

15 Huang, J., Niu, J.: 'A fairness-based and adaptive user grouping and subcarrier allocation algorithm for grouped MC-CDMA systems'. Proc. IEEE GLOBECOM, November 2006, pp. 1-5

16 Wang, Y., Shen, J., Zhang, P., Liu, S., Liu, Y.: 'Optimized power control and resource allocation in grouped MC-CDMA systems'. Proc. IEEE ICC, May 2008, pp. 4785-4789

17 Cai, X., Zhou, S., Giannakis, G.: 'Group-orthogonal multicarrier CDMA', IEEE Trans. Commun., 2004, 52, (1), pp. 90-99

18 Han, C., Harrold, T., Armour, S.: 'Green radio: radio techniques to enable energy-efficient wireless networks', IEEE Commun. Mag., 2011, 49, (6), pp. 46-54

19 Proakis, J.: 'Digital communications' (McGraw-Hill, New York, 1995)

20 3rd Generation Partnership Project (3GPP); Technical specification group radio access network; Radio transmission and reception (Release 7)

21 Rappaport, T.S.: 'Wireless communications' (Prentice-Hall, 2002)

22 Fehske, A., Richter, F., Fettweis, G.: 'Energy efficiency improvements through micro sites in cellular mobile radio networks'. Proc. Second Workshop on Green Communications, 2009 